\title{
PROPERTIES OF ENDOMORPHISM RINGS OF MODULES AND THEIR DUALS
}

\author{
SOUMAYA MAKDISSI KHURI
}

\begin{abstract}
Let ${ }_{R} M$ be a nonsingular left $R$-module whose Morita context is nondegenerate, let $B=$ End $_{R} M$ and let $M^{*}=\operatorname{Hom}_{R}(M, R)$. We show that $B$ is left (right) strongly modular if and only if any element of $B$ which has zero kernel in ${ }_{R} M\left(M_{R}^{*}\right)$ has essential image in ${ }_{R} M\left(M_{R}^{*}\right)$, and that $B$ is a left (right) Utumi ring if and only if every submodule ${ }_{R} U$ of ${ }_{R} M\left(U_{R}^{*}\right.$ of $\left.M_{R}^{*}\right)$ such that $U^{\perp}=0\left({ }^{\perp} U^{*}=0\right)$ is essential in ${ }_{R} M\left(M_{R}^{*}\right)$.
\end{abstract}

1. Introduction. Let ${ }_{R} M$ be a left $R$-module whose standard Morita context is nondegenerate (see Definition 1); let $B=\operatorname{End}_{R} M$ be the ring of $R$-endomorphisms of ${ }_{R} M$ and let $M^{*}=\operatorname{Hom}_{R}(M, R)$ be its dual module. Then $B$ is left nonsingular if and only if ${ }_{R} M$ is nonsingular (i.e. $M$ satisfies the following: any $m \in M$ with essential annihilator in $R$ must be zero), and $B$ is right nonsingular if and only if $M_{R}^{*}$ satisfies the following condition: If $U_{R}^{*}$ is an essential submodule of $M_{R}^{*}$ then the annihilator of $U^{*}$ in $B$ must be zero (Proposition 5). This condition certainly holds if $M_{R}^{*}$ is nonsingular. Of course, just as for ${ }_{R} M, M_{R}^{*}$ is nonsingular if and only if End ${ }_{R} M^{*}$ is right nonsingular. Our concern, however, is with $B$, which is in general - for example for a nonfinitely generated ${ }_{R} M-$ a proper subring of $\operatorname{End}_{R} M^{*}$; hence a condition on ${ }_{R} M$ which is equivalent to a certain left property of $B$ is not expected to be equivalent to the same right property of $B$ when it is reflected in $M_{R}^{*}$. In this paper, we investigate this situation and try to pick out some left-right properties of $B$ which are symmetrically, or almost symmetrically, represented on ${ }_{R} M$ and $M_{R}^{*}$. For example, we find that $B$ is left strongly modular if and only if any element of $B$ which has zero kernel in ${ }_{R} M$ has essential image in ${ }_{R} M$, while $B$ is right strongly modular if and only if any element of $B$ which has zero kernel in $M_{R}^{*}$ has essential image in $M_{R}^{*}$ (Theorem 3); and we find that $B$ is a left Utumi ring if and only if every submodule ${ }_{R} U$ of ${ }_{R} M$ such that $U^{\perp}=0$ is essential in ${ }_{R} M$, while $B$ is a right Utumi ring if and only if every submodule $U_{R}^{*}$ of $M_{R}^{*}$ such that ${ }^{\perp} U^{*}=0$ is essential in $M_{R}^{*}$ (Theorem 7). These conditions naturally raise the general question of how $B$ sits in End $M_{R}^{*}$, a question which we do not treat in this paper, but which we expect to investigate in a future article.

Received by the editors February 4, 1985.

1980 Mathematics Subject Classification. Primary 16A08, 16A65.

$\mathrm{Kev}$ words and phrases. Endomorphism rings, nonsingular modules and rings, nondegenerate Morita contexts, maximal quotient rings, Utumi rings. 
2. Preliminaries. The left and right annihilators in $B$ of a subset $K$ of $B$ will be denoted by $\mathscr{L}(K)$ and $\mathscr{R}(K)$, respectively. The notation $l_{M}(K), r_{M^{*}}(K), r_{B}(U)$, $l_{B}\left(U^{*}\right)$ will be used for the annihilators in $M$ of $K \subseteq B$ in $M^{*}$ of $K \subseteq B$, in $B$ of $U \subseteq M$ and in $B$ of $U^{*} \subseteq M^{*}$, respectively. Also, for ${ }_{R} U \subseteq{ }_{R} M$ and $U_{R}^{*} \subseteq M_{R}^{*}$, we will use: $I_{B}(U)=\{b \in B: M b \subseteq U\}$ and $I_{B}\left(U^{*}\right)=\left\{b \in B: b M^{*} \subseteq U^{*}\right\}$. The notation ${ }_{R} U \subset{ }_{R}^{e} M$ will be used to indicate that $U$ is an essential $R$-submodule of $M$, i.e. $U$ intersects nontrivially every nonzero $R$-submodule of $M$. Recall that ${ }_{R} M$ is said to be nonsingular in case, for $m \in M, l_{R}(m) \subset{ }_{R} R \Rightarrow m=0 ; B$ is said to be left (right) nonsingular if ${ }_{B} B\left(B_{B}\right)$ is nonsingular.

We recall the following definition and proposition from [4]:

Definition 1. Let $(R, M, N, S)$ be a Morita context; that is, let ${ }_{R} M_{S}$ and ${ }_{S} N_{R}$ be bimodules with an $R-R$ bimodule homomorphism (, ): $M \otimes{ }_{S} N \rightarrow R$ and an $S-S$ bimodule homomorphism [, ]: $N \otimes_{R} M \rightarrow S$ satisfying

$$
m_{1}\left[n_{1}, m_{2}\right]=\left(m_{1}, n_{1}\right) m_{2} \text { and } n_{1}\left(m_{1}, n_{2}\right)=\left[n_{1}, m_{1}\right] n_{2}
$$

for all $m_{1}, m_{2} \in M$ and $n_{1}, n_{2} \in N$.

Then $(R, M, N, S)$ is said to be nondegenerate if and only if the four modules ${ }_{R} M$, $M_{S},{ }_{S} N, N_{R}$ and the two pairings are faithful (the latter meaning that $(m, N)=0$ implies $m=0$, and three analogous implications).

This is equivalent to the eight natural maps associated with the Morita context being injective (for example, two of these maps are: $m \mapsto(m,-)$ and $r \mapsto(n \mapsto n r)$ $\in \operatorname{End}\left({ }_{s} N\right)$, for $m \in M, n \in N$ and $\left.r \in R\right)$. The standard context $\left(R, M, M^{*}, B\right)$ of a module ${ }_{R} M$ is nondegenerate if and only if ${ }_{R} M$ is torsionless and faithful and the right annihilator of trace $\left({ }_{R} M\right)$ is zero. We shall call such a module-i.e. one whose standard context is nondegenerate-a nondegenerate module, for brevity.

Proposition 1 [4, Proposition 14]. If the context $(R, M, N, S)$ is nondegenerate, and if one of ${ }_{R} R,{ }_{R} M,{ }_{S} N,{ }_{S} S$ is nonsingular, then all of them are nonsingular.

Henceforth, unless otherwise indicated, let ${ }_{R} M$ be a nondegenerate, nonsingular left $R$-module. Then, by the preceding, ${ }_{R} M, M_{B},{ }_{B} M^{*}, M_{R}^{*}$ and the two pairings are faithful, and ${ }_{R} R,{ }_{R} M^{*}$ and ${ }_{B} B$ are nonsingular. (, ) and [, ] will denote the pairings associated with the standard context for ${ }_{R} M$, i.e. ( , ) is defined by $(m, f)=m f$ for $m \in M$ and $f \in M^{*}$, and $[f, m]$ is defined by $m_{1}[f, m]=\left(m_{1}, f\right) m$ for all $m$, $m_{1} \in M$ and $f \in M^{*}$.

If ${ }_{R} U$ is a submodule of ${ }_{R} M$ then $\left[M^{*}, U\right]$ indicates the left ideal of $B$ : $\left[M^{*}, U\right]=\left\{\sum_{i=1}^{s}\left[m_{i}^{*}, u_{i}\right]: m_{i}^{*} \in M^{*}, u_{i} \in U_{i}\right\}$, and similarly for $\left[U^{*}, M\right]$ where $U_{R}^{*}$ is a submodule of $M_{R}^{*}$. Also, $U^{\perp}=\left\{m^{*} \in M^{*}:\left(U, m^{*}\right)=0\right\}$ and ${ }^{\perp} U^{*}=\{m \in M$ : $\left.\left(m, U^{*}\right)=0\right\}$.

The well-known fact that, for a nonsingular module ${ }_{R} M$, any $R$-homomorphism $f$, to ${ }_{R} M$ from any other $R$-module, which has essential kernel is zero, will be used repeatedly without comment.

The following lemma will be useful to us in the sequel.

Lemma 2. For $K \subseteq B, \mathscr{L}(K)=I_{B} l_{M}(K)=l_{B}\left(K M^{*}\right)$, and $\mathscr{R}(K)=r_{B}(M K)=$ $I_{B}^{*} r_{M^{*}}(K)$. 
ProOF.

$$
\begin{aligned}
& b \in \mathscr{L}(K) \Leftrightarrow b K=0 \Leftrightarrow M b K=0 \Leftrightarrow M b \subseteq l_{M}(K) \Leftrightarrow b \in I_{B} l_{M}(K) \\
& b \in \mathscr{L}(K) \Leftrightarrow b K=0 \Leftrightarrow b K M^{*}=0 \Leftrightarrow b \in l_{B}\left(K M^{*}\right) \\
& b \in \mathscr{R}(K) \Leftrightarrow K b=0 \Leftrightarrow M K b=0 \Leftrightarrow b \in r_{B}(M K) \\
& b \in \mathscr{R}(K) \Leftrightarrow K b=0 \Leftrightarrow K b M^{*}=0 \Leftrightarrow b M^{*} \subseteq r_{M^{*}}(K) \Leftrightarrow b \in I_{B}^{*} r_{M^{*}}(K) .
\end{aligned}
$$

3. Strongly modular and Utumi endomorphism rings. In [2], a Baer ${ }^{*}$-ring $B$ is called strongly modular in case, for all $b$ in $B, \mathscr{R}(b)=0$ implies that $b B$ is essential in $B$. Because of the involution, the definition is left-right symmetric. In the absence of an involution, call a ring $B$ left strongly modular if, for $b \in B, \mathscr{L}(b)=0 \Rightarrow B b$ $\subset{ }_{B}{ }_{B} B$, and right strongly modular if $\mathscr{R}(b)=0 \Rightarrow b B \subset{ }^{e} B_{B}$. It turns out that the properties of left and right strong modularity of $B=\operatorname{End}_{R} M$ are equivalent to almost symmetric conditions on ${ }_{R} M$ and $M_{R}^{*}$.

THEOREM 3. (i) $B$ is left strongly modular if and only if, for each $b \in B$, $l_{M}(b)=0 \Rightarrow M b \subset{ }_{R}^{e} M$;

(ii) $B$ is right strongly modular if and only if, for each $b \in B, r_{M^{*}}(b)=0 \Rightarrow b M^{*}$ $\subset^{e} M_{R}^{*}$.

Proof. By comparing the definition of left strong modularity with the condition on ${ }_{R} M$ in (i), it is easily seen that (i) will follow as soon as we show that " $\mathscr{L}(b)=0$ " is equivalent to " $l_{M}(b)=0$ " and that " $B b \subset{ }_{B}^{e} B$ " is equivalent to " $M b \subset{ }_{R}^{e} M$ "; these equivalences will be proved in Lemma 4 which follows. Similarly, (ii) will follow once we show, in Lemma 4 , that " $\mathscr{R}(b)=0$ " is equivalent to " $r_{M^{*}}(b)=0$ " and that " $b B \subset{ }^{e} B_{B}$ " is equivalent to " $b M^{*} \subset{ }^{e} M_{R}^{*}$ ".

LemMA 4. (i) For any subset $K$ of $B, \mathscr{L}(K)=0$ if and only if $l_{M}(K)=0$ and $\mathscr{R}(K)=0$ if and only if $r_{M^{*}}(K)=0$.

(ii) For any left ideal ${ }_{B} H$ of $B,{ }_{B} H \subset{ }^{e}{ }_{B} B$ if and only if $M H \subset{ }^{e}{ }_{R} M$; and for any right ideal $J_{B}$ of $B, J_{B} \subset{ }^{e} B_{B}$ if and only if $J M^{*} \subset{ }^{e} M_{R}^{*}$.

Proof. (i) Let $K \subseteq B$ and consider the submodule $l_{M}(K)$ of ${ }_{R} M$. If $l_{M}(K) \neq 0$, let $0 \neq m \in l_{M}(K)$; then, by nondegeneracy, there is $m^{*} \in M^{*}$ such that $\left[m^{*}, m\right] \neq$ 0 . Then, since $M_{B}$ is faithful, $0 \neq M\left[m^{*}, m\right]=\left(M, m^{*}\right) m \subseteq R m \subseteq l_{M}(K)$; that is, $0 \neq\left[m^{*}, m\right] \in I_{B} l_{M}(K)$. Hence, $l_{M}(K)=0$ if and only if $\mathscr{L}(K)=I_{B} l_{M}(K)=0$.

Similarly, if $0 \neq m^{*} \in r_{M^{*}}(K)$, then nondegeneracy gives $m \in M$ such that $\left[m^{*}, m\right] \neq 0$, and since ${ }_{B} M^{*}$ is faithful, $\left[m^{*}, m\right]$ is a nonzero element of $I_{B}^{*} r_{M^{*}}(K)$; hence $r_{M^{*}}(K)=0$ if and only if $\mathscr{R}(K)=I_{B}^{*} r_{M^{*}}(K)=0$.

(ii) Let ${ }_{B} H$ be an essential left ideal of $B$ and let $0 \neq m \in M$. Then $\left[M^{*}, m\right] \cap H$ $\neq 0$, and, since $M_{B}$ is faithful,

$0 \neq M\left(\left[M^{*}, m\right] \cap H\right) \subseteq M\left[M^{*}, m\right] \cap M H=\left(M, M^{*}\right) m \cap M H \subseteq R m \cap M H$, proving that $M H \subset{ }_{R}^{e} M$.

Conversely, assume that $M H \subset{ }_{R}^{e} M$ for some left ideal ${ }_{B} H$ of $B$ and let $0 \neq c \in B$. Then, since $M_{B}$ is faithful, $M c \neq 0$, and hence $M c \cap M H \neq 0$. By nondegeneracy,

$$
0 \neq\left[M^{*}, M c \cap M H\right] \subseteq\left[M^{*}, M c\right] \cap\left[M^{*}, M H\right] \subseteq B c \cap\left[M^{*}, M H\right] .
$$


This shows that $\left[M^{*}, M H\right] \subset{ }_{B} B$, and hence, since $\left[M^{*}, M H\right] \subseteq{ }_{B} H$, we have ${ }_{B} H \subset{ }_{B}^{e} B$.

Similarly, if $J$ is an essential right ideal of $B$ and $0 \neq m^{*} \in M^{*}$, then, by nondegeneracy, $\left[m^{*}, M\right] \neq 0$, hence $\left[m^{*}, M\right] \cap J \neq 0$. Since $M_{B}^{*}$ is faithful, this implies

$$
\begin{aligned}
0 & \neq\left(\left[m^{*}, M\right] \cap J\right) M^{*} \subseteq\left[m^{*}, M\right] M^{*} \cap J M^{*} \\
& =m^{*}\left(M, M^{*}\right) \cap J M^{*} \subseteq m^{*} R \cap J M^{*}
\end{aligned}
$$

so $J M^{*} \subset^{e} M_{R}^{*}$.

Conversely, if $J M^{*} \subset{ }^{e} M_{R}^{*}$ for some right ideal $J_{B}$ of $B$, and $0 \neq c \in B$, then $J M^{*} \cap c M^{*} \neq 0$ and $\left[J M^{*} \cap c M^{*}, M\right] \neq 0$ by nondegeneracy; hence,

$$
0 \neq\left[J M^{*} \cap c M^{*}, M\right] \subseteq\left[J M^{*}, M\right] \cap\left[c M^{*}, M\right] \subseteq\left[J M^{*}, M\right] \cap c B .
$$

This implies that $\left[J M^{*}, M\right] \subset{ }^{e} B_{B}$, and hence, since $\left[J M^{*}, M\right] \subseteq J$, we have $J_{B} \subset{ }^{e}$ $B_{B}$.

REMARKS. 1. One property of nondegenerate modules that can be deduced from the proof of Lemma 4 is that $I_{B}(U)=0$ if and only if $U=0$ for a submodule ${ }_{R} U$ of ${ }_{R} M$, and similarly for $U_{R}^{*} \subseteq M_{R}^{*}$.

2. In the proof of Lemma 4(ii), we have shown that ${ }_{R} U \subset{ }_{R}^{e} M \Rightarrow\left[M^{*}, U\right] \subset{ }_{B}^{e} B$ and $U_{R}^{*} \subset{ }^{e} M_{R}^{*} \Rightarrow\left[U^{*}, M\right] \subset{ }^{e} B_{B}$.

Aside from completing the proof of Theorem 3, Lemma 4 is also useful in giving a condition on $M_{R}^{*}$ which is equivalent to right nonsingularity of $B$, as in the next result.

Proposition 5. B is right nonsingular if and only if, for any submodule $U_{R}^{*}$ of $M_{R}^{*}$, $U_{R}^{*} \subset{ }^{e} M_{R}^{*} \Rightarrow l_{B}\left(U^{*}\right)=0$.

Proof. It was shown in [3, Proposition 1] that, under our present hypotheses, $B$ is right nonsingular if and only if, for any submodule ${ }_{R} U$ of ${ }_{R} M, r_{B}(U) \subset{ }^{e} B_{B} \Rightarrow U=$ 0 .

Assume that $B$ is right nonsingular and suppose that $U_{R}^{*} \subset{ }^{e} M_{R}^{*}$; then, as noted in Remark 2 above, $\left[U^{*}, M\right] \subset \subset^{e} B_{B}$. We have $\left(M l_{B}\left(U^{*}\right)\right)\left[U^{*}, M\right]=$ $\left(M l_{B}\left(U^{*}\right), U^{*}\right) M=0$; therefore $\left[U^{*}, M\right] \subseteq r_{B}\left(M l_{B}\left(U^{*}\right)\right)$, which implies $r_{B}\left(M l_{B}\left(U^{*}\right)\right) \subset{ }^{e} B_{B}$. Hence, by [3, Proposition 1], since $B$ is right nonsingular, this implies that $M l_{B}\left(U^{*}\right)=0$; hence, since $M_{B}$ is faithful, we have $l_{B}\left(U^{*}\right)=0$.

Conversely, assume that $U_{R}^{*} \subset{ }^{e} M_{R}^{*}$ implies $l_{B}\left(U^{*}\right)=0$. Suppose that ${ }_{R} U$ is a submodule of ${ }_{R} M$ such that $r_{B}(U) \subset{ }^{e} B_{B}$. Then, by Lemma 4(ii), $U_{R}^{*}=r_{B}(U) M^{*}$ $\subset^{e} M_{R}^{*}$. Hence, by hypothesis, $l_{B}\left(r_{B}(U) M^{*}\right)=0$. But $I_{B}(U) \subseteq l_{B}\left(r_{B}(U) M^{*}\right)$ since, always, $I_{B}(U) r_{B}(U)=0$; hence $I_{B}(U)=0$, which, by nondegeneracy (see Remark 1 ), implies that $U=0$, completing the proof.

A ring $B$ is said to be a left Utumi ring in case, for any left ideal ${ }_{B} H$ of $B$, $\mathscr{R}\left({ }_{B} H\right)=0 \Rightarrow{ }_{B} H \subset{ }_{B}^{e} B ; B$ is called a right Utumi ring if, for any right ideal $J_{B}$ of $B, \mathscr{L}\left(J_{B}\right)=0 \Rightarrow J_{B} \subset{ }^{e} B_{B}$. In [2], it is shown that a strongly modular Baer *-ring is left and right Utumi [2, Theorem 2.3]. In our situation, i.e. for $B=\operatorname{End}_{R} M$, where ${ }_{R} M$ is nondegenerate and nonsingular, it is easily shown that a left and right strongly modular Baer ring satisfies the Utumi conditions for principal left and right 
ideals. In fact, $B$ need not be a Baer ring to show this; rather, left and right nonsingularity of $B$ is sufficient, with left and right strong modularity, in order to obtain the Utumi conditions for principal ideals, as can be seen in Proposition 6. For the full Utumi conditions on $B$, however, we can give, in Theorem 7 , conditions on ${ }_{R} M$ and $M_{R}^{*}$ which appear quite symmetrical.

Proposition 6. If ${ }_{R} M$ is such that $B=\operatorname{End}_{R} M$ is a left and right nonsingular, left and right strongly modular ring, then

(i) $\mathscr{L}(b B)=0 \Rightarrow b B \subset{ }^{e} B_{B}$, and (ii) $\mathscr{R}(B b)=0 \Rightarrow B b \subset{ }_{B}^{e} B$.

PROOF. (i)

$$
\begin{aligned}
\mathscr{L}(b B)=\mathscr{L}(b)=0 & \Rightarrow B b \subset{ }^{e}{ }_{B} B \quad \text { since } B \text { is left strongly modular, } \\
& \Rightarrow \mathscr{R}(B b)=0 \quad \text { since } B \text { is left nonsingular, } \\
& \Rightarrow b B \subset{ }^{e} B_{B} \quad \text { since } B \text { is right strongly modular. }
\end{aligned}
$$

(ii)

$$
\begin{aligned}
\mathscr{R}(B b)=\mathscr{R}(b)=0 & \Rightarrow b B \subset{ }^{e} B_{B} \quad \text { since } B \text { is right strongly modular, } \\
& \Rightarrow \mathscr{L}(b B)=0 \quad \text { since } B \text { is right nonsingular, } \\
& \Rightarrow B b \subset{ }^{e}{ }_{B} B \quad \text { since } B \text { is left strongly modular. }
\end{aligned}
$$

For our last result, ${ }_{R} M$ is assumed to satisfy the standing hypothesis, i.e. ${ }_{R} M$ is nonsingular and nondegenerate.

THEOREM 7. (i) $B=\operatorname{End}_{R} M$ is a left Utumi ring if and only if, for any submodule ${ }_{R} U$ of ${ }_{R} M, U^{\perp}=0 \Rightarrow{ }_{R} U \subset{ }_{R}^{e} M$; and (ii) $B=\operatorname{End}_{R} M$ is a right Utumi ring if and only if, for any submodule $U_{R}^{*}$ of $M_{R}^{*},{ }^{\perp} U^{*}=0 \Rightarrow U_{R}^{*} \subset{ }^{e} M_{R}^{*}$.

Proof. (i) Assume that $B$ is a left Utumi ring; then, by [3, Lemma 3], we have, for any submodule ${ }_{R} X$ of ${ }_{R} M, r_{B}\left({ }_{R} X\right)=0 \Rightarrow{ }_{R} X \subset{ }_{R}^{e} M$. Let ${ }_{R} U$ be a submodule of ${ }_{R} M$ such that $U^{\perp}=0$. Then $b \in r_{B}(U) \Rightarrow U b=0 \Rightarrow\left(U, b m^{*}\right)=\left(U b, m^{*}\right)=0$, for each $m^{*} \in M^{*}, \Rightarrow b m^{*}=0$ since $U^{\perp}=0$; but this means $b M^{*}=0$, therefore, since ${ }_{B} M^{*}$ is faithful, $b=0$. Hence $r_{B}(U)=0$, which implies, since $B$ is left Utumi, that ${ }_{R} U \subset{ }^{e} M$.

Conversely, assume that ${ }_{R} U^{\perp}=0 \Rightarrow{ }_{R} U \subset{ }^{e}{ }_{R} M$ for every ${ }_{R} U \subseteq{ }_{R} M$. Let ${ }_{B} H$ be a left ideal of $B$ with $\mathscr{R}\left({ }_{B} H\right)=0$. If $\left(M H, m^{*}\right)=0$, then $\left(M, H m^{*}\right)=0$, which implies $H m^{*}=0$ by nondegeneracy. Then $\left[H m^{*}, M\right]=0$, i.e. $H\left[m^{*}, M\right]=0$, which implies $\left[m^{*}, M\right]=0$ since $\mathscr{R}(H)=0$. Again by nondegeneracy, $\left[m^{*}, M\right]=0 \Rightarrow m^{*}$ $=0$. Hence, we have shown that $\left(M H, m^{*}\right)=0 \Rightarrow m^{*}=0$, i.e. $(M H)^{\perp}=0$, which by hypothesis implies that $M H \subset{ }_{R}^{e} M$. Now, by Lemma 4(ii), this gives ${ }_{B} H \subset{ }_{B} B$, and $B$ is left Utumi.

(ii) Assume that $B$ is a right Utumi ring. Let $U_{R}^{*}$ be a submodule of $M_{R}^{*}$ such that ${ }^{\perp} U^{*}=0$. Consider the right ideal $\left[U^{*}, M\right]$ of $B$. If $m\left[U^{*}, M\right]=0$, then $\left(m, U^{*}\right) M$ $=0$, hence, since ${ }_{R} M$ is faithful, $\left(m, U^{*}\right)=0$, which gives $m=0$ since ${ }^{\perp} U^{*}=0$. Therefore, $l_{M}\left(\left[U^{*}, M\right]\right)=0$, hence, by Lemma $4(\mathrm{i}), \mathscr{L}\left(\left[U^{*}, M\right]\right)=0$, which implies that $\left[U^{*}, M\right] \subset{ }^{e} B_{B}$ since $B$ is right Utumi. Then, by Lemma 4(ii), $\left[U^{*}, M\right] M^{*} \subset^{e}$ $M_{R}^{*}$. But $\left[U^{*}, M\right] M^{*} \subseteq U^{*}$, hence $U_{R}^{*} \subset{ }^{e} M_{R}^{*}$, and we have shown that ${ }^{\perp} U^{*}=0$ implies $U_{R}^{*} \subset{ }^{e} M_{R}^{*}$. 
Conversely, assume that ${ }^{\perp} U^{*}=0 \Rightarrow U_{R}^{*} \subset{ }^{e} M_{R}^{*}$ for any submodule $U_{R}^{*}$ of $M_{R}^{*}$. Let $J_{B}$ be a right ideal of $B$ such that $\mathscr{L}\left(J_{B}\right)=0$. Then, by Lemma $4(\mathrm{i}), l_{M}(J)=0$; hence, if $\left(m, J M^{*}\right)=0$, then $m J=0$ by nondegeneracy, and $m=0$ since $l_{M}(J)=0$. Thus, ${ }^{\perp}\left(J M^{*}\right)=0$, which, by hypothesis, implies that $J M^{*} \subset{ }^{e} M_{R}^{*}$. Finally, by Lemma 4(ii), $J M^{*} \subset{ }^{e} M_{R}^{*} \Rightarrow J_{B} \subset{ }^{e} B_{B}$, completing the proof that $B$ is right Utumi.

Remarks. 1. The nondegeneracy condition on ${ }_{R} M$ cannot be deleted from the hypothesis of Theorem 7, as we shall see in the following example.

First recall that a CS module is one in which every complement (= essentially closed) submodule is a direct summand, with a ring $R$ being left or right CS whenever ${ }_{R} R$ or $R_{R}$ is a CS module. In [1], an example is given of a nonsingular, projective CS module $P$ whose endomorphism ring, $B=$ End $P$, is not left CS (Example 3.3 in [1]). We will show that, for such a $P$, the condition " $U^{\perp}=0 \Rightarrow U$ $\subset^{e} P$, for any submodule $U$ of $P$ " of Theorem 7(i) does hold, and yet $B=$ End $P$ is not left Utumi, the reason being that the nondegeneracy condition does not hold in $P$.

Assume that $U^{\perp}=0$ for a submodule $U$ of $P$. Then, $b \in r_{B}(U) \Rightarrow U b=0 \Rightarrow$ $\left(U, b P^{*}\right)=\left(U b, P^{*}\right)=0 \Rightarrow b P^{*}=0$ since $U^{\perp}=0$, and this last gives $b=0$ since ${ }_{B} P^{*}$ is faithful, which shows that $r_{B}(U)=0$. Now, since $P$ is a CS module, the essential-closure, $U^{e}$, of $U$ is a direct summand in $P$, say $P=U^{e} \oplus V$, and there is an idempotent $b \in B$ such that $U^{e} b=0$ and $v b=v$ for $v \in V$; then $r_{B}(U)=0$ implies that $b=0$, so $V=0$ and $U \subset \subset^{e} P$.

To see that $B$ is not left Utumi, recall first that a ring is left nonsingular, left CS if and only if it is Baer and left Utumi (cf. e.g. [1, Theorem 2.1]); thus, since $B$ is not left CS, it will suffice to show that $B$ is Baer: Let $J$ be any subset of $B$, then the essential closure, $(P J)^{e}$, of $P J$ is a direct summand in $P$ since $P$ is $\mathrm{CS}$, say $P=(P J)^{e} \oplus U$; then, letting $e$ be the idempotent in $B$ with ker $e=(P J)^{e}$, we have $\mathscr{R}(J)=r_{B}(P J)=r_{B}\left((P J)^{e}\right)=e B$, which proves that $B$ is a Baer ring.

Finally, to see that nondegeneracy of $P$ does not hold, we remark that (a) $P$ nondegenerate $\Rightarrow I_{B}(U) \neq 0$ for every nonzero submodule $U$ of $P$, as noted in Remark 1 following Theorem 3; and (b) " $I_{B}(U) \neq 0$ for every $0 \neq U \subseteq P$ " does not hold in $P$, because by Lemma 3 of [3] a nonsingular module with this property has a left Utumi endomorphism ring if and only if " $r_{B}(U)=0 \Rightarrow U \subset{ }^{e} P$ ", and we have just shown this last to be true in $P$, whereas $B$ is not left Utumi.

2. In the special case when the nondegenerate, nonsingular ${ }_{R} M$ is ${ }_{R} R$, it is easy to see that the conditions in Theorem 7 are precisely the Utumi conditions for a left and right nonsingular $R$. We verify this for the left Utumi condition, by noting that " $U^{\perp}=0$ " becomes just " $r_{B}(U)=0$ " or " $\mathscr{R}(I)=0$ " for $I$ a left ideal in $B$. For, in this case, $B=\operatorname{End}\left({ }_{R} R\right) \cong R$; thus, if ${ }_{R} U={ }_{R} I$ is a left ideal in $R$, then $I^{\perp}=0 \Rightarrow$ $r_{B}(I)=0: b \in r_{B}(I) \Rightarrow I b=0 \Rightarrow\left(I, b R^{*}\right)=\left(I b, R^{*}\right)=0 \Rightarrow b R^{*}=0$ since $I^{\perp}=$ $0, \Rightarrow b=0$ since ${ }_{B} R^{*}$ is faithful; and, conversely, $r_{B}(I)=0 \Rightarrow I^{\perp}=0: r^{*} \in I^{\perp} \Rightarrow$ $\left(I, r^{*}\right)=0 \Rightarrow I r^{*} r=\left(I, r^{*} r\right)=0$ for each $r \in R$, and this last implies that $r^{*} r=0$ for each $r \in R$, when we consider $r^{*} r$ as being in $R \cong B$ and use the fact that $r_{B}(I)=0$; finally, $r^{*} R=0 \Rightarrow r^{*}=0$ since $R_{R}^{*}$ is faithful. 


\section{REFERENCES}

1. A. W. Chatters and S. M. Khuri, Endomorphism rings of modules over non-singular CS rings, J. London Math. Soc. (2) 21 (1980), 434-444.

2. D. Handelman, Coordinatization applied to finite Baer ${ }^{*}$-rings, Trans. Amer. Math. Soc. 235 (1978), 1-34.

3. S. M. Khuri, Modules whose endomorphism rings have isomorphic maximal left and right quotient rings, Proc. Amer. Math. Soc. 85 (1982), 161-164.

4. B. J. Müller, The quotient category of a Morita context, J. Algebra 28 (1974), 389-407.

Department of Mathematics, Yale University, New Haven, Connecticut 06520 